\title{
Fourier-Based Diffractive Shearing Interferometer for Wavefront Sensing
}

\author{
Jason H. Karp, Trevor K. Chan and Joseph E. Ford \\ Department of Electrical and Computer Engineering, University of California, San Diego \\ 9500 Gilman Drive., La Jolla, CA 92093-0409, USA \\ phone: 858822 4406, fax: 858534 1225, jkarp@ucsd.edu, jeford@ucsd.edu
}

\begin{abstract}
We propose a simple and compact wavefront sensor using a phase transmission grating attached to a CMOS image sensor. We present experimental results using a binary phase grating. An adaptive grating displayed by a phase modulator can extend sensor dynamic range.

\section{Introduction}

Shack-Hartmann $(\mathrm{SH})$ sensors are compact and integrated wavefront sensors which consist of fixed lenslets above a sensor plane. Local tilt in the incident wavefront produces a lateral shift of the focal spot formed by each lenslet. The limitations of $\mathrm{SH}$ sensors come from the limited dynamic range of the detector, and the choice of lenslet focal length which forces a tradeoff between resolution and dynamic range.

Diffractive shearing (DS) interferometry is an alternate wavefront sensor that uses a grating to generate overlapping diffraction orders at the sensor plane. DS interferometers require at least partial coherence (coherence length larger than the shifted path difference) but many applications use laser or narrow band LED illumination. A gradient phase measurement is embedded in an interference fringe pattern, distributing the signal over the entire sensor thereby making better use of the detector's dynamic range. A fixed grating pitch again forces a tradeoff between resolution and dynamic range, but efficient diffraction gratings can be displayed using a liquid crystal (LC) spatial light modulator (SLM), allowing the sensor to adapt in response to the input wavefront, either globally, with a single spatial frequency, or locally, generating a partially compensated fringe pattern. Here we propose an integrated diffractive shearing wavefront sensor incorporating a LC modulator attached directly over a CMOS image sensor. This paper provides early results where we demonstrate the basic geometry using a fixed diffraction grating and synthesize the processing techniques to evaluate its performance.
\end{abstract}

\section{Experimental Setup}

A binary phase transmission grating provides high efficiency diffraction into the $\pm 1^{\text {st }}$ orders while the diffraction angle causes a tilt between the wavefront replicas. The linear tilt gives rise to a sinusoidal fringe pattern that acts as a carrier for gradient information [1]. We perform carrier demodulation using the Fourier transform [2] and reconstruction via modal estimation [3].

We cemented the grating to the CMOS sensor surface and coherent illumination generates a carrier sinusoid with $\sim 3$ pixels per fringe. Though within the Nyquist limit, the speckle-reduced modulation contrast leaves the spectral peaks indiscernible. Since a longer period grating was unavailable, we incorporated a $4 \mathrm{~F}$ relay imaging system with magnification to increase the fringe period at the cost of reduced spectral separation.

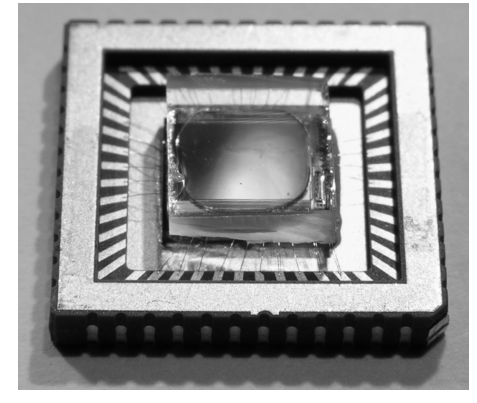

(a)

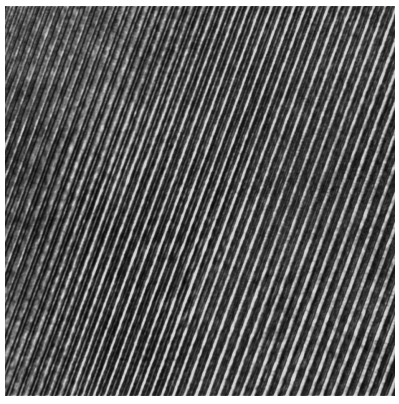

(b)

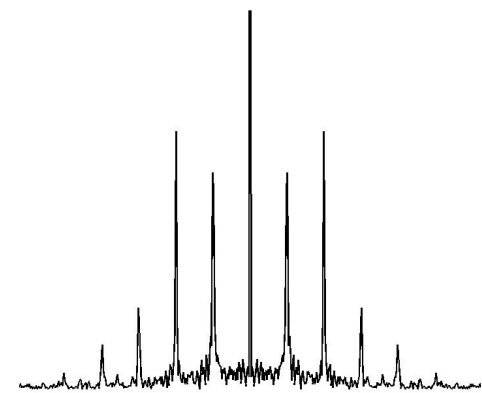

(c)

Fig 1: phase grating mounted to a CMOS sensor (a), interference containing spherical phase curvature captured using a 4F relay (b), 1D Fourier transform of figure 1-b (c) 


\section{Performance}

We used a $100 \mathrm{~mm}$ plano-convex lens as the input phase object to test the performance of the system. The grating is spaced from the object to create a 0.1 shearing ratio that can be adjusted via translation. After demodulation, a linearly increasing differential phase measurement is computed with $2 \pi$ phase wrapping. Variations as large as $14 \%$ are observed in the gradient indicating the consequences of reduced carrier separation. After integration with modal estimation and polynomial fitting, the spherical profile of the lens is reconstructed. For comparison, we measured the object using an interference microscope. A slightly shorter radius of curvature is computed with an overall difference of $9.2 \%$ between the two techniques.

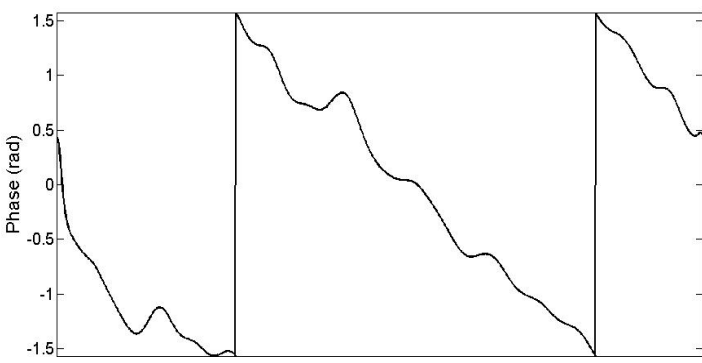

(a)

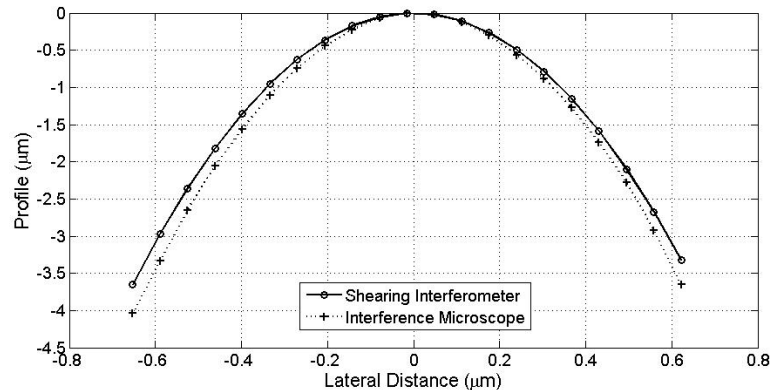

(b)

Fig 2: phase-wrapped gradient profile (a), reconstructed phase profile from diffractive shearing

\section{Wavefront Adaptation}

The sensitivity of shearing interferometers largely depends on the profile of the incoming wavefront. Steep gradients should be computed using small shearing ratios to maximize the dynamic range of the sensor and avoid potential phase wraps between points of overlap, while slowly varying profiles require larger shear distances to overlap portions of the wavefront with greater phase differences. An arbitrary wavefront may contain characteristic from both regimes supporting the use of a SLM to display gratings with different periods to measure individual portions of the profile with high sensitivity. We will present mathematical analysis to address the relationship between shearing ratio and reconstruction accuracy for various wavefront profiles and SNR levels.

\section{Conclusion}

We integrated a fixed diffraction grating and image sensor together to create a compact wavefront sensor without the need for additional lenses or spatial filters. Transmission-type spatial light modulators are powerful additions to this design and are to be incorporated in subsequent analyses. The improved usage of dynamic range and adaptability of diffractive shearing may surpass the limitations inherent to Shack-Hartmann wavefront sensors.

\section{Acknowledgements}

This work was supported by DARPA as part of the Montage Project. The authors also thank Digital Optics Corporation for the donation of sample binary phase gratings

\section{References}

[1] M. Takeda, "Fourier-Transform method of fringe-pattern analysis for computer-based topography and interferometry," J. Opt. Soc. Am. 72, 156-160 (1982).

[2] S. Nicola \& P. Ferraro, "Fourier transform method of fringe analysis for moiré interferometry," J. Opt. A: Pure Appl. Opt. 2 228-233 (2000).

[3] K. Freischlad \& C. Koliopoulos, "Modal estimation of a wavefront from difference measurements using the discrete Fourier transform," Opt. Soc. Am. Vol. 3 1852-1861 (1986).

[4] N. Lindlein, J. Pfund, \& J. Schwider, "Expansion of the dynamic range of Shack-Hartmann sensor by using astigmatic microlenses," Opt. Eng. Vol. 39, No. 8, 2220-2225 (2000). 